\title{
Bondad para las prácticas evaluativas en la plataforma virtual Moodle durante la enseñanza de posgrado universitaria
}

Kindnees for Assess practices on the virtual Moodle platform during university postgraduate teaching

Recibido: marzo 04 de 2021 | Revisado: abril 12 de 2021 | Aceptado: mayo 04 de 2021

\author{
Rosalvina Campos Pérez ${ }^{\mathrm{I}}$ \\ Luis Celi SaAvedra ${ }^{2}$ \\ George Argota Pérez ${ }^{3}$ \\ Rina Ma. Állvarez Becerra ${ }^{4}$ \\ Ramiro M. Yallico CalmetT ${ }^{5}$ \\ VIOLETA Romero Carrión ${ }^{6}$
}

\begin{abstract}
Resumen
El desarrollo de habilidades en la enseñanza universitaria se relaciona con los procesos de construcción e interacciones de conocimientos prácticos. El objetivo del estudio fue describir la bondad para las prácticas evaluativas en la plataforma virtual Moodle durante la enseñanza de posgrado universitaria. El estudio se realizó entre febrero y marzo de 2021 donde se reconoció como bondades de prácticas evaluativas de la plataforma Moodle, el chat, cuestionario, foro y la tarea. Las bondades prácticas de la plataforma virtual Moodle mejoran la autonomía de los estudiantes, pues no solo se arriesgan a mostrar sus habilidades, sino que descubren nuevas oportunidades a partir de los conocimientos expuestos por otros estudiantes. Se concluye que la plataforma virtual Moodle genera bondades prácticas donde se permite mediante la comprensión de elementos indicativos mejorar el rendimiento de los estudiantes, además, de significar la plataforma virtual Moodle enfoque innovador enseñanza híbrida con la enseñanza presencial, pues a través de sus actividades prácticas finalmente, se satisfacen habilidades.
\end{abstract}

Palabras clave: colaboración, enseñanza-aprendizaje, proceso educativo, universidad, virtualidad

\begin{abstract}
The development of skills in university teaching is related to the processes of construction and interactions of practical knowledge. The objective of the study was to describe the goodness for evaluative practices in the Moodle virtual platform during university postgraduate teaching. The study was conducted between February and March 2021 where it was recognized as benefits of evaluative practices of the Moodle platform, the chat, questionnaire, forum and task. The practical benefits of the virtual platform Moodle improve the autonomy of students, since they not only risk showing their skills, but also discover new opportunities based on the knowledge presented by other students. It is concluded that the virtual platform Moodle generates practical benefits where it is allowed through the understanding of indicative elements to improve the performance of students, in addition, the Moodle virtual platform means an innovative approach to hybrid teaching with face-to-face teaching, because through its practical activities, skills are finally satisfied.
\end{abstract}

Key words: collaboration, teaching-learning, educational process, university, virtuality

(C) Los autores. Este artículo es publicado por la Revista Campus de la Facultad de Ingeniería y Arquitectura de la Universidad de San Martín de Porres. Este artículo se distribuye en los términos de la Licencia Creative Commons Atribución No-comercial - Compartir-Igual 4.0 Internacional (https://creativecommons.org/licenses/ CC-BY), que permite el uso no comercial, distribución y reproducción en cualquier medio siempre que la obra original sea debidamente citada. Para uso comercial contactar a: revistacampus@usmp.pe. 


\section{Introducción}

Ante la declaración de la Organización Mundial de la Salud (OMS) sobre la pandemia de COVID-19 el 30 de enero de 2020 (Sohrabi et al., 2020) numerosos cambios en la sectorización global se han producidos como, por ejemplo salud (Peng et al., 2020, Holshue et al., 2020), agricultura (Bochtis et al., 2020), industria manufacturera (Knieps, 2020), energía (Mohamed, 2020), sistemas socioeconómicos (Bucketal., 2020; Nicola et al., 2020), donde los modelos de competencia para la comunicación entre sectores incluso comienzan a pensar en migrar hacia lo virtual (Argota \& Argota, 2021). De igual modo, el sector educacional no es una excepción, y donde se observa algunas interrupciones de ciclos académicos (Panesar et al., 2020).

A consideración de los autores, la falta de elementos combinados hace preocupante la enseñanza y el aprendizaje en los estudiantes de pregrado y posgrado en las universidades, pues no solo es la disponibilidad de medios ordenadores (incluyendo laptops) o tabletas para la conexión en línea, capacidad de transmisión, inestabilidad en el suministro de energía eléctrica, ausencia de educación durante la comunicación virtual, sino que existe falta de preparación previa por parte, de la generalidad de docentes. Sin embargo, debe reconocerse que el desafío ante lo nuevo y por necesidad se impone por cuanto, la búsqueda de vías y aceptación en lo particular, relacionada con algunas plataformas que deben promover la continuidad durante la enseñanza.

En tal sentido, la enseñanza virtual genera motivaciones para el desarrollo del aprendizaje y la formación de los estudiantes, pues exige mostrar competencias reales desde el uso de recursos educativos digitales, tecnologías interactivas; así como el trabajo independiente todo lo cual, garantiza desde la calificación profesional, satisfacer las necesidades sociales en la modernidad (Oproiu, 2015; Shchitov et al., 2015).

Una de las plataformas libre de código abierto, aprendizaje en línea y de reconocimiento potencial, es la Moodle (Modular Object-Oriented Dynamic Learning Environment) creada por Martin Dougiamas y ampliamente utilizada en la enseñanza de segundas lenguas (Gómez et al., 2009; Lopera, 2012). El modelo pedagógico de la plataforma Moodle es socio-constructivista y donde se muestra el aprendizaje desde la construcción del conocimiento intra e inter colaboración de la comunidad (Savio, 2020). Asimismo, la plataforma Moodle sirve como repositorio de materiales didácticos y mediante el control remoto promueve el logro del proceso enseñanza y aprendizaje orientado a objetivos modulares (Costa et al., 2012; Guinaldo et al., 2013; Barge \& Londhe, 2014). En general, Moodle significa "entorno de aprendizaje dinámico orientado a objetos modulares" y es una de las plataformas en línea más populares que se utiliza con fines educativos (Badia et al., 2019; De Medio et al., 2020), y en comparación con otras plataformas como Schoology o ATutor. Moodle destaca por su diseño personalizable y una amplia variedad de herramientas que se pueden usar de manera efectiva para crear varias oportunidades de aprendizajes síncronas como asincrónicas (Krouska et al., 2018).

El aprendizaje colaborativo mediante ordenadores permite un nuevo paradigma 
que relaciona las principales teóricas del propio aprendizaje con los recursos tecnológicos considerándose el entorno de desarrollo social y cultural (Casillas et al., 2017), la retroalimentación continua (Dooley \& Bamford, 2018) y luego, excelentes interrelaciones colaborativas para la formación del aprendizaje satisfactorio (Chen \& Kuo, 2019).

No existe duda que el rol de los docentes universitarios resulta más activo en los escenarios de colaboración mediante las plataformas virtuales, pues los resultados a esperar, dependerán en general, de criterios prácticos a demostrarse (van
Leeuwen \& Janssen, 2019).

El objetivo del estudio fue describir la bondad para las prácticas evaluativas en la plataforma virtual Moodle durante la enseñanza de los cursos de posgrado universitarios.

\section{Métodos}

El estudio se realizó entre febrero y marzo de 2021 donde se consideró la plataforma de enseñanza virtual Moodle. Al considerarse, ańadir una actividad o un recurso, el sistema visualiza diversas opciones (Figura 1):

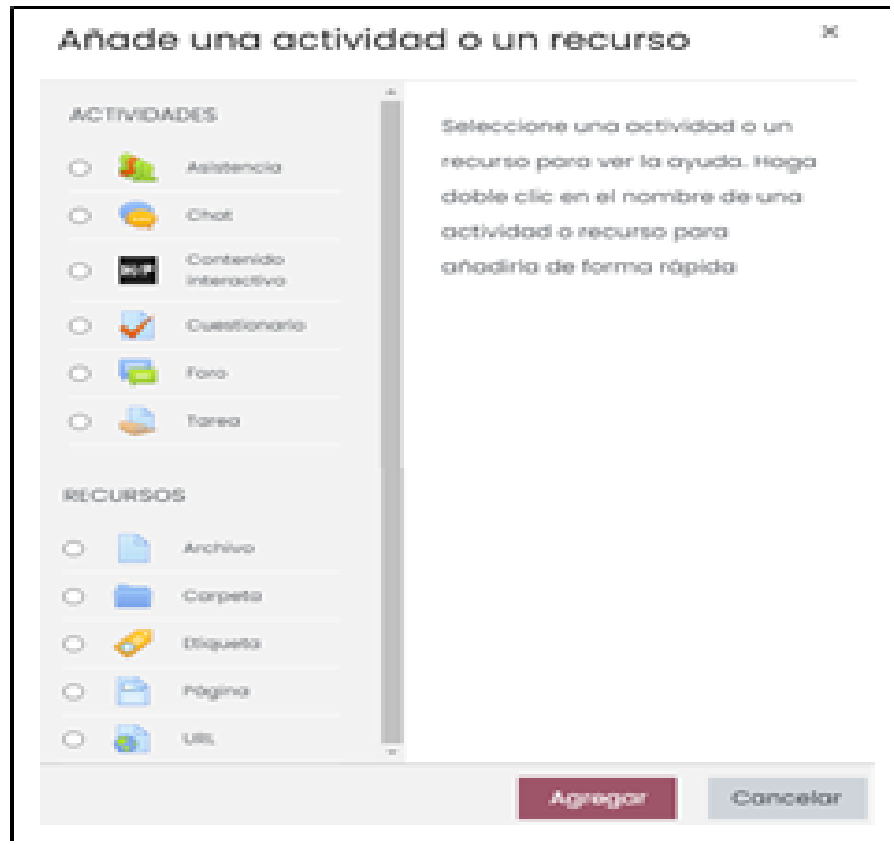

Figura 1. Visualización de opciones / actividades / recursos / plataforma Moodle

Se reconoció como bondades de prácticas evaluativas de la plataforma Moodle el chat, cuestionario, foro y la tarea.

Se considera como aspecto ético de la investigación, prescindir toda construcción improcedente en la construcción de la información científica, parafraseo inapropiado y la ausencia de citas de autores.

\section{Resultados y Discusión}

Se muestra el criterio de reporte al seleccionar cualquiera de las opciones de bondades prácticas de la plataforma virtual Moodle donde la hermenéutica del docente según los objetivos a proponerse, determinarán el reconocimiento de las habilidades durante el proceso de enseñanza y aprendizaje (Tabla 1). 
Rosalvina Campos Pérez - Luis Celi Saavedra - George Argota Pérez - Rina Ma. Álvarez Becerra Ramiro M. Yallico Calmett - Violeta Romero Carrión

Tabla 1

Actividades / reporte / plataforma virtual Moodle

\begin{tabular}{|c|c|}
\hline Actividades & Reporte \\
\hline Chat & $\begin{array}{l}\text { Permite la discusión en formato texto de manera sincrónica en tiempo real. La misma } \\
\text { puede ser, puntual o repetirse a la misma hora cada día o cada semana. Las sesiones } \\
\text { de chat se guardan y pueden hacerse públicas para que todos las vean o limitadas a los } \\
\text { usuarios con permiso para ver los registros de sesiones del chat. } \\
\text { Los chats son, especialmente, útiles cuando un grupo no tiene posibilidad de reunirse } \\
\text { físicamente para conversar donde se visualiza cara-cara. Asimismo, los chats permiten: } \\
\text { - Reuniones programadas donde se permite el compartir experiencias. } \\
\text { - La comunicación con el docente y actualizarse en caso de ausencia. } \\
\text { - Conocer y mejorar las relaciones mediante el inicio o la continuidad del uso de } \\
\text { - las redes. } \\
\text { - Cacilitar las preguntas y respuestas. }\end{array}$ \\
\hline Cuestionario & $\begin{array}{l}\text { Permite diseñar y plantear cuestionarios con preguntas tipo opción múltiple, } \\
\text { verdadero/falso, coincidencia, respuesta corta y respuesta numérica. } \\
\text { Admite resolver varias veces las preguntas ordenadas o seleccionadas aleatoriamente } \\
\text { del banco de preguntas. Se puede establecer un tiempo límite. } \\
\text { Cada intento se califica automáticamente, con la excepción de las preguntas de tipo } \\
\text { "ensayo" y el resultado se guarda en el libro de calificaciones. } \\
\text { Los cuestionarios pueden usarse para realizar: } \\
\text { - Exámenes de prácticas y de curso } \\
\text { - Mini Test para tareas de lectura o al final de un tema } \\
\text { - Ofrecer información inmediata sobre el rendimiento. }\end{array}$ \\
\hline Foro & $\begin{array}{l}\text { Permite las discusiones asincrónicas. } \\
\text { Hay varios tipos de foro: estándar, de sección única para la discusión, foro de } \\
\text { preguntas y respuestas. } \\
\text { El profesor puede permitir que se adjunten archivos a las aportaciones al foro. } \\
\text { Los mensajes en el foro pueden ser evaluados tanto por los docentes como por los } \\
\text { propios estudiantes (evaluación por pares). } \\
\text { Existen múltiples usos de los foros: } \\
\text { - Espacio social de presentación e intercambios } \\
\text { - Comunicación del curso, sus contenidos y lecturas } \\
\text { - Comunicación oculta entre docentes } \\
\text { - Centro de ayuda y facilitación de consejos } \\
\text { - Compartir actividades complementarias }\end{array}$ \\
\hline Tarea & $\begin{array}{l}\text { Permite revisar, valorar, evaluar, calificar y retroalimentar el aprendizaje de los } \\
\text { alumnos. } \\
\text { Facilita la presentación de contenidos digitales. } \\
\text { Acepta las tareas individuales y posibilita vincularse con las restantes. } \\
\text { Las tareas pueden ser calificadas usando una escala numérica o una escala personalizada } \\
\text { o usando métodos de calificación complejos como rúbricas. }\end{array}$ \\
\hline
\end{tabular}

Ante los reportes de la plataforma virtual Moodle, se permite la exploración de diferentes opciones de aprendizaje mediante sus bondades prácticas 
donde los estudiantes al utilizar las instrucciones de esta herramienta digital en línea, construyen y evalúan sus propios conocimientos. De igual modo, no se interrumpen actividades programadas y se crean múltiples oportunidades intercambiables. Aunque la práctica académica en línea es un tema de investigación, durante las últimas tres décadas, con la presencia del COVID-19, existió muy poco tiempo para la preparación de manera eficiente del proceso enseñanza y aprendizaje. Sin embargo, aprovechar la bondad de las plataformas virtuales como Moodle, indica nuevos desafíos en la enseñanza (Glassey \& Magalhães, 2020).

Desde las bondades prácticas de la plataforma virtual Moodle se mejora la autonomía de los estudiantes, pues no solo arriesgan mostrar sus habilidades, sino que descubren nuevas oportunidades, a partir de los conocimientos expuestos por otros estudiantes (Seifan et al., 2020; Granjo \& Rasteiro, 2020). Asimismo, debe indicarse con toda responsabilidad que entre las probables desventajas que al menos pudiera existir, está precisamente la sobrecarga mental y con lo cual agotaría al estudiante ante las disímiles instrucciones que pueden ser utilizadas por el docente (Pekdağ, 2020).

Entre las principales limitaciones del estudio se encontró no evaluar las prácticas de los estudiantes desde la plataforma virtual Moodle lo que facilitaría la hermenéutica del proceso de enseñanza y aprendizaje.

Se concluye que, la bondad para las prácticas evaluativas desde la plataforma virtual Moodle durante la enseñanza de posgrado universitaria permite la comprensión de elementos indicativos y ante la contextualización de la enseñanza en línea, los estudiantes mejoran sus rendimientos y con ello, satisfacen la creación de habilidades. Así como lo indica (Nancy et al., 2020), el enfoque innovador de la pedagogía de la enseñanza avanzada que orienta a mejorar el proceso de enseñanza y aprendizaje determina que se produzca una enseñanza híbrida entre el aprendizaje electrónico y la enseñanza presencial donde el diseño de preguntas, la evaluación práctica de estudiantes, el proceso de retroalimentación cognoscitiva y la metodología de la investigación son pilares fundamentales en el éxito de uso con la plataforma virtual Moodle.

\section{Referencias}

Argota, P.Y. \& Argota, P.G. (2021). Modelo de consultoría digital en la comunicación social con el sistema empresarial productivo. Cátedra Villarreal; 8, 167-172. https://doi. org/10.24039/cv202082974

Badia, A., Martín, D. \& Gomez, M. (2019). Teachers' perceptions of the use of moodle activities and their learning impact in secondary education. Technology Knowledge and Learning; 24(3), 483-499. https://doi.org/10.1007/s10758018-9354-3

Barge, P. \& Londhe, B.R. (2014). From Teaching, Learning to Assessment: MOODLE Experience at B'School in India. Procedia Economics and Finance; 11, 857-865. https://doi.10.1016/ s2212-5671(14)00249-4 
Bochtis, D., Benos, L., Lampridi, M., Marinoudi, V., Pearson, S. \& Sørensen, C.G. (2020). Agricultural Workforce Crisis in Light of the COVID-19 Pandemic. Sustainability; 12, 1-13; http:// doi. $10.3390 /$ su 12198212

Buck, T., Arnold, M., Chazan, G. \& Cookson, C. (2020). Coronavirus declared a pandemic as fears of economic crisis mount. https:// www.ft.com/content/d72f1e546396-11 ea-b3f3-fe4680ea68b5

Casillas, M.S., Cabezas, G.M. \& Hernández, M.A. (2017). Construcción de conocimiento colaborativo mediado tecnológicamente: aportaciones teóricas desde el análisis de prácticas educativas. Teoría de la Educación. Revista Interuniversitaria; 29, 6186. https://doi.org/10.14201/ teoredu2926186

Chen, C.M. \& Kuo, C.H. (2019). An optimized group formation scheme to promote collaborative problem-based learning. Computers and Education; 133, 94-115. https://doi.org/10.1016/j. compedu.2019.01.011

Costa, C., Alvelos, H. \& Teixeira, L. (2012). The use of moodle e-learning platform: A Study in a Portuguese University. Procedia Technology; 5, 334-343. http:// doi.10.1016/j.protcy.2012.09.037

De Medio, C., Limongelli, C., Sciarrone, F. \& Temperini, M. (2020). MoodleREC: A recommendation system for creating courses using the moodle e-learning platform. Computers in Human Behavior; 104, 1-33. https://doi. org/10.1016/j.chb.2019.106168

Dooley, L.M. \& Bamford, N.J. (2018). Peer feedback on collaborative learning activities in veterinary education. Veterinary Sciences; 5, 90. https://doi.org/10.3390/ vetsci5040090

Glassey, J. \& Magalhães, F.D. (2020). Virtual labs - love them or hate them, they are likely to be used more in the future. Educ. Chem. Eng., 33, 76-77. https://doi. org/10.1016/j.ece.2020.07.005

Gómez, I., Hernández, G.E. \& Rico, G.M. (2009). Moodle en la enseñanza presencial y mixta del inglés en contextos universitarios. RIED. Revista Iberoamericana de Educación a Distancia; 12(1), 69193.

Granjo, J.F.O. \& Rasteiro, M.G. (2020). Enhancing the autonomy of students in chemical engineering education with labvirtual platform. Educ. Chem. Eng; 31, 2128. https://doi.org/10.1016/j. ece.2020.03.002

Guinaldo, M., de la Torre, L., Heradio, R. \& Dormido, S. (2013). A Virtual and Remote Control Laboratory in Moodle: The Ball and Beam System. IFAC Proceedings Volumes; 46, 72 77. http://doi.10.3182/201308283-uk-2039.00021

Holshue, M.L., DeBolt, C. \& Lindquist, S. (2020). First case of 2019 
novel coronavirus in the United States New England Journal of Medicine; 382, 929-936. http:// doi.10.1056/NEJMoa2001191

Knieps, S. (2020). Will COVID-19 turn Germany's export-oriented economy into a weakness? https:// www.euractiv.com/section/ economy-jobs/news/

Krouska, A., Troussas, C. \& Virvou, M. (2018). Comparing LMS and CMS platforms supporting social e-learning in higher education. In 2017 8th International Conference on Information, Intelligence, Systems and Applications; 1-6. https://doi. org/10.1109/IISA.2017.8316408

Lopera, M.S.A. (2012). El uso de la plataforma educativa MOODLE en un curso de competencia lectora en inglés como Lengua Extranjera (ILE). Núcleo, 24(29), 79-103

Mohamed, T. (2020). There is no escape: Stocks, oil, and bitcoin plunge as US lawmakers fight over coronavirus rescue package. https://markets. businessinsider.com/news/stocks/ no-escape-stocks-oil-bitcoinplungesenate-argues-coronavirusbill-2020-3-1029021850

Nancy, W., Parimala, A. \& Livingston, L. M.M. (2020). Advanced teaching pedagogy as innovative approach in modern education system. Procedia Computer Science; 172, 382-388. http://doi:10.1016/j. procs.2020.05.059

Nicola, M., Alsafi, Z., Sohrabi, C., Kerwan, A., Al-Jabir, A.,
Iosifidis, C, Agha, M. \& Agha, R. (2020). The socio-economic implications of the coronavirus pandemic (COVID-19): A review International Journal of Surgery; 78, 185-193. https://doi. org/10.1016/j.ijsu.2020.04.018

Oproiu, G.C. (2015). A Study about Using E-learning Platform (Moodle) in University Teaching Process. Procedia-Social and Behavioral Sciences; 180, 426432. http://doi.10.1016/j. sbspro.2015.02.140

Panesar, K., Dodson, T., Lynch, J., Bryson, C.C., Chew, L. \& Dillon, J. (2020). Evolution of COVID-19 guidelines for University of Washington oral and maxillofacial surgery patient care. https://doi. org/10.1016/j.joms.2020.04.034

Pekdağ, P. (2020). Video-based instruction on safety rules in the chemistry laboratory: its effect on student achievement. Chem. Educ. Res. Pract; 21, 953-968. http://dx.doi.org/10.1039/ D0RP00088D

Peng, X., Xu, X. \& Li. Y. (2020). Transmission routes of 2019$\mathrm{nCoV}$ and controls in dental practice. International Journal of Oral Science; 399, 1-7. https://doi.org/10.1016/j. neucom.2020.02.064

Savio, K. (2020). La plataforma Moodle en la alfabetización académica: Uso del aula virtual en un taller de lectura y escritura. Páginas de Educación, 13(1), 1-18. 
https://dx.doi.org/10.22235/ pe.v13i1.1923

Seifan, M., Robertson, N. \& Berenjian, A. (2020). Use of virtual learning to increase key laboratory skills and essential non-cognitive characteristics. Educ. Chem. Eng, 33, 66-75. https://doi. org/10.1016/j.ece.2020.07.006

Shchitov, A.G., Shchitova, O.G., Shchitova, D.A., Stasinska, P. \& Chieu, D.T.C. (2015). Features of the Learning Modular System Moodle Use in Teaching the Russian Language to Russian and Foreign Students at an Institution of Higher Education. Procedia Social and Behavioral Sciences; 215,
170-175. http://doi.10.1016/j. sbspro.2015.11.613

Sohrabi, C., Alsafi, Z., O’Neill, N., Kerwan, A. \& Al-Jabir, A. (2020). World Health Organization declares global emergency: A review of the 2019 novel coronavirus (COVID-19). International Journal of Surgery; 76, 71-76. https://doi. org/10.1016/j.ijsu.2020.02.034

Van Leeuwen, A. \& Janssen, J. (2019). A Systematic Review of Teacher Guidance During Collaborative Learning in Primary and Secondary Education. Educational Research Review; 27, 71-89. http:// doi.10.1016/j.edurev.2019.02.001 